\title{
Cortico-cortical connectivity between right parietal and bilateral primary motor cortices during imagined and observed actions: a combinedTMS/tDCS study
}

\author{
Matteo Feurra, Giovanni Bianco, Nicola R. Polizzotto, Iglis Innocenti, Alessandro Rossi and Simone Rossi*
}

Sezione Neurologia e Neurofisiologia Clinica, Dipartimento di Neuroscienze, Azienda Ospedaliera Universitaria Senese, Policlinico le Scotte, Siena, Italy

Edited by:

Giacomo Koch, Fondazione Santa Lucia IRCCS, Italy

Reviewed by:

Ryota Kanai, University College

London, UK

Miguel Fernandez-Del-Olmo,

Universidade da Coruña, Spain

*Correspondence:

Simone Rossi, Sezione Neurologia e Neurofisiologia Clinica, Dipartimento di Neuroscienze, Policlinico Le Scotte,

Viale Bracci, I-53100 Siena, Italy.

e-mail: rossisimo@unisi.it
Previous transcranial magnetic stimulation (TMS) studies showed functional connections between the parietal cortex (PC) and the primary motor cortex (M1) during tasks of different reaching-to-grasp movements. Here, we tested whether the same network is involved in cognitive processes such as imagined or observed actions. Single pulse TMS of the right and left M1 during rest and during a motor imagery and an action observation task (i.e., an index-thumb pinch grip in both cases) was used to measure corticospinal excitability changes before and after conditioning of the right PC by 10 min of cathodal, anodal, or sham transcranial direct current stimulation (tDCS). Corticospinal excitability was indexed by the size of motorevoked potentials (MEPs) from the contralateral first dorsal interosseous (FDI; target) and abductor digiti minimi muscle (control) muscles. Results showed selective ipsilateral effects on the M1 excitability, exclusively for motor imagery processes: anodal tDCS enhanced the MEPs' size from the FDI muscle, whereas cathodal tDCS decreased it. Only cathodal tDCS impacted corticospinal facilitation induced by action observation. Sham stimulation was always uneffective. These results suggest that motor imagery, differently from action observation, is sustained by a strictly ipsilateral parieto-motor cortex circuits. Results might have implication for neuromodulatory rehabilitative purposes.

Keywords:TMS, tDCS, motor imagery, action observation, connectivity, M1, right PC

\section{INTRODUCTION}

The parietal cortex (PC) plays an important role in some of the principal brain functions such as attentional, memory, and perceptual learning processes of auditory, tactile, and visual stimuli (Colby and Goldberg, 1999; Cohen and Andersen, 2002; Rossi et al., 2006; Law and Gold, 2008; Zimmer, 2008; Giovannelli et al., 2010), suggesting that it represents an associative area which cross-modally integrates multi-sensory information (Calvert, 2001). Recently, by a new twin-coil paired-pulse transcranial magnetic stimulation (TMS) conditioning method developed by Koch et al. (2007), which makes possible to causally address functional connectivity between two different cortical areas, it has been demonstrated in vivo that the right $\mathrm{PC}$ and its sub-regions such as posterior parietal cortex (PPC), and different portions of the intra-parietal sulcus (IPS), have functional connections with the ipsilateral, or even the contralateral primary motor cortex (M1; Koch et al., 2007, 2008, 2009). Such a connectivity may be relevant in bilateral hand coordination, movement planning, and grasping actions (Grefkes and Fink, 2005; Koch et al., 2009). In addition, the interconnection between $\mathrm{PC}$ and $\mathrm{M} 1$ could also play a role in higher-order cognitive motor functions, such as motor imagery and action observation. Indeed, evidence exists for a widely distributed cortical network underlying action observation and motor imagery processes, which in part overlaps (Decety et al., 1994, 1997; Nelissen et al., 2005), but definitely encompasses the parietal lobe (Rizzolatti and Craighero, 2004; Fogassi and Luppino, 2005).
Motor imagery is a covert cognitive process defined as the ability of a subject to mentally perform movement of her/his own body-part without actually moving that part, and without even subliminally tensing the engaged muscles (Jeannerod, 1995, 2001; Lotze and Cohen, 2006). The neural network involved during motor imagery includes the same brain areas which are recruited during planning, online controlling and executing of a movement, such as the primary, premotor, supplementary, inferior, superior, PPC, cerebellum, and basal ganglia (Decety et al., 1994; Parsons et al., 1995; Schnitzler et al., 1997; Hanakawa et al., 2003; Dechent et al., 2004; Fleming et al., 2010). Action observation, defined as the perception of the actions of others, is a cognitive task which produces the activation of the motor system that simulates what would happen if the observer himself would execute the observed action without any motor output (Fadiga et al., 1995; Grafton et al., 1996; Rizzolatti et al., 2001), a process likely correlated to the mirror-system activity (Rizzolatti and Craighero, 2004). Action observation could induce the observer's motor system to resonate, thereby representing the functional substrate underlying action recognition (Rizzolatti and Fadiga, 1998).

In the present study, we aimed to causally verify whether motor imagery and/or action observation involve parietal-motor cortex connections. In order to test this hypothesis, we used transcranial direct current stimulation (tDCS) as a neuromodulatory technique to produce an off-line conditioning of the right PC while subjects had to perform a motor imagery and an action observation task engaging the same muscle groups. Although the TMS conditioning 
method developed by Koch et al. (2007), is efficient enough in studying the functional connectivity between two different cortical areas, tDCS could bring clear cut advantages for the current experimental purpose. Indeed, there are evidences that cortical neurons may respond to static electrical fields applied by electrodes placed on the scalp by changing their membrane potential in a selective manner depending by the electric current direction (Wagner et al., 2007). Whereas the excitability of a target cortical area increases with anodal polarization, it decreases with cathodal one (Priori et al., 1998; Nitsche and Paulus, 2000; Wassermann and Grafman, 2005), with after-effects outlasting the tDCS application. Moreover tDCS has been proved to induce reliable effects on corticospinal excitability of the human motor cortex (Nitsche and Paulus, 2001; Nitsche et al., 2003b,c; Antal et al., 2008), as well as the premotor cortex during motor imagery (Quartarone et al., 2004). Here, a conditioning electrical current to PC was administered by using three different experimental conditions: cathodal, anodal, and sham (placebo) tDCS in a randomized blocked fashion design. Corticospinal output was indexed through the analysis of a test response (i.e., motor-evoked potentials, MEPs) evoked by single pulse TMS of the right (ipsilateral) and left (contralateral) primary motor cortex (M1).

\section{MATERIALS AND METHODS PARTICIPANTS}

Eleven healthy right-handed, not naïve to TMS, participants (seven males and four females, aged 20-35) took part in the experiment. They all reported to be fully right-handed and with no history of neurological or psychiatric problems. They also denied the assumption of drugs or alcohol in the days preceding the experiments. The experiment was approved by the local Ethical Committee. Subjects were fully informed of the nature of the research and signed an informed consent to participate.

\section{TMS AND EMG}

Stimulation was delivered through a Magstim Super Rapid stimulator with four external boosters with a maximum output of approximately $2 \mathrm{~T}$ (Magstim, Whitland, UK) connected with a standard figure-of-eight 70-mm coil. The coil was held by hand tangential to the scalp, with the handle pointing backward and laterally, angled at $45^{\circ}$ from the midline sagittal axis of the participants' head, with its handle pointing backward. It was positioned on the region of the left or right hemiscalp triggering MEPs from the contralateral examined hand muscles with the minimal threshold (hot spot) which was defined in agreement to international standards (Rossi et al., 2009). The hot spot was marked on the scalp, in order to allow the same coil positioning during the experiments. The TMS intensity was adjusted to produce fairly stable basal MEPs of $600-800 \mu \mathrm{V}$ in the right first dorsal interosseous (FDI; prime mover in the imagined and observed actions). MEPs from the abductor digiti minimi muscle (ADM) were also simultaneously recorded. Such TMS intensity corresponded to about 110-115\% of the individual motor threshold. $\mathrm{Ag}-\mathrm{AgCl}$ adhesive electrodes were applied over the target muscles in a belly-tendon bipolar montage, with the active electrode placed on the motor point of each muscle. MEPs were recorded by a four-channel electromyograph (Phasis, EBNeuro), with a bandpass filter of $20 \mathrm{~Hz}-2 \mathrm{KHz}$, sampled at $20 \mathrm{KHz}$, with a gain range of $0.1-1.5 \mathrm{mV}$. A total time epoch of $200 \mathrm{~ms}$ was analyzed in each trial, on which the first $100 \mathrm{~ms}$ were serving as pre-trigger analysis time, in order to monitor and exclude those trials that might be contaminated by unwanted background EMG activity. By taking into account the complex design (number of conditions, number of TMS targets, pre-tDCS, and post-tDCS application) of the experiment, six MEPs centered on the median latency value for each condition were collected. This relatively low number of trials/condition is in line with a previous study (Rossi et al., 2008) and was adopted here in order to get an optimal compromise among the length of experiment, the amount of collected data and the tiredness of subjects throughout the experiment. Moreover, an accurate monitoring of motor responses, allowed us to minimize the probability that MEPs belonging to different neural pools (i.e., with onset latency shorter than $1.5 \mathrm{~ms}$ from the mean) could be included in the analysis. Indeed, it is known that a latency shortening occurs even in case of subliminal voluntary contraction occurring in the $300 \mathrm{~ms}$ preceding the instant of TMS (Starr et al., 1988; Rossi et al., 2008). Every TMS pulse was spaced $10-15 \mathrm{~s}$ from the previous one.

\section{TRANSCRANIAL DIRECT CURRENT STIMULATION}

Transcranial direct current stimulation was delivered by a battery driven, constant current stimulator (Eldith DC-Stimulator by Neuro Conn, Germany) using a pair of surface saline-soaked sponge electrodes. A $5 \mathrm{~cm} \times 7 \mathrm{~cm}$ electrode was used both for the site of stimulation and for the reference. During the experiment, the target electrode was placed over the right PC (according to the international 10-20 system) while the reference over the ipsilateral shoulder (Cogiamanian et al., 2007; Vandermeeren et al., 2010). The current flow was applied for $10 \mathrm{~min}$ with an intensity of $1500 \mu \mathrm{A}$ (Ardolino et al., 2005). The current density at the stimulation electrode corresponded to $21.4 \mathrm{~mA} / \mathrm{cm} 2$, thus below $25 \mathrm{~mA} / \mathrm{cm}^{2}$, in order to do not cause any brain tissue damage (McCreery et al., 1990; Nitsche et al., 2003a). Sham stimulation consisted in just a few seconds of stimulation, in order to induce subjects to feel an itching sensation that is usually felt during the rising up of real tDCS and it goes to diminish during the time course of the experiment. This short-lasting sham tDCS do not produce any after-effect (Nitsche and Paulus, 2000).

\section{EXPERIMENTAL DESIGN}

The experiment consisted in three main sessions. Cathodal, anodal, and sham stimulation were performed separately for each session, spaced at least 5 days apart. The order of the sessions was randomized and counterbalanced across subjects (Figure 1).

For each session, subjects underwent to MEPs measurements from the right and left FDI muscles during a resting, a motor imagery and an action observation task (always a index-thumb pinch grip, in which the FDI acts as prime mover of the action) that were performed prior and after tDCS. As a control, MEPs were also simultaneously recorded by the ADM muscle, which shares the cortical representation with the FDI muscle but was not involved in the imagined or observed task.

During the resting task, complete muscular and mental relaxation of every subject was requested. This condition was then referred as the "baseline." For motor imagery task, imagery of right 


\section{Experimental Task}

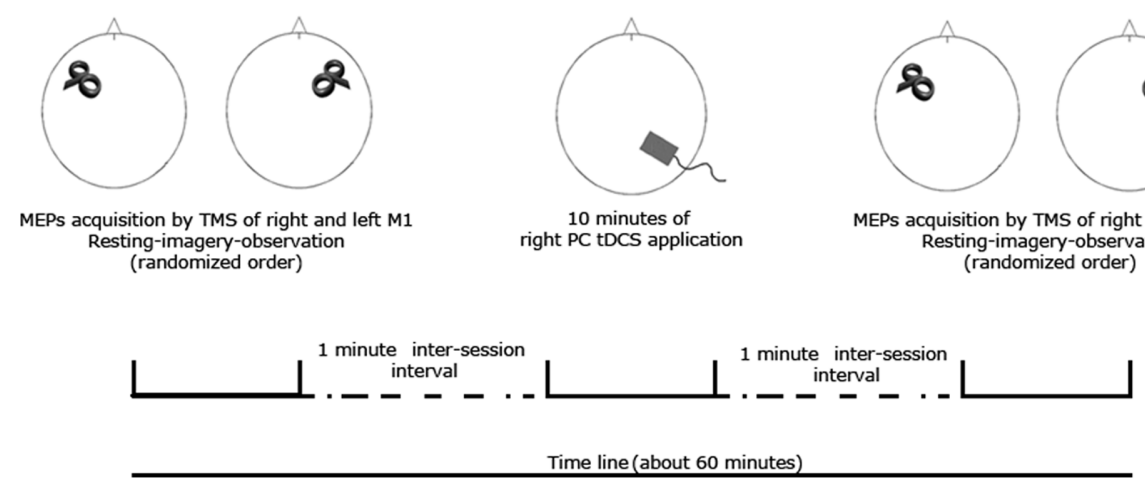

FIGURE 1 | Experimental task. Subjects were submitted to a resting, motor imagery and an action observation task previously and after each tDCS session (anodal, cathodal, sham) spaced 5-7 days apart. Corticospinal excitability was measured by TMS of left and right primary motor cortex. The order of tDCS and task and TMS target (left and right $\mathrm{M} 1$ ) conditions was fully randomized.

or left index finger abduction (mental activation of the FDI, while the $\mathrm{ADM}$ was at rest) was requested; simulated movements were performed 2-3s after the experimenter verbal command, therefore outside from the usual reaction time of a subject (Rossini et al., 1999). This condition was subsequently referred as the "motor imagery." Of note, during this task, subjects were asked to adopt visual motor imagery (subject sees him/her self performing the movement as from a third person perspective) respect to the kinesthetic motor imagery strategy (subject has the feeling to perform the movement; Ruby and Decety, 2003), in order to better compare the motor imagery task with the passive action observation task. During the action observation task, the subjects were asked to observe the experimenter's right or left hand (depending by the subject's hand monitored by the EMG, thus contralateral to the right or left M1 TMS stimulation) actually doing the same index-thumb pinch grip that was requested for the motor imagery task. This condition was referred as "action observation."

All the experimental conditions, including left and right M1 single pulse TMS was randomized for each session across subjects.

\section{DATA ANALYSIS}

Peak-to-peak maximal amplitude of each MEP was calculated offline; then, six artifact-free couple of FDI/ADM MEPs/condition were averaged. Preliminary repeated measures ANOVAs were run in order to check for any cortical excitability changes between the pre- and post-tDCS MEPs at rest. Results did not show any significant effects (see Appendix). Thus, pre- and post-tDCS changes of the motor imagery and action observation average MEP size were analyzed as the percentage of the mean peak-to-peak amplitude of the basal MEP measured during the resting task (baseline condition; Rossini et al., 1999) recorded previously and after each tDCS session.

Mean MEPs size of FDI and ADM activity was evaluated by two separate four-way ANOVA, with two levels of "task" factor (motor imagery, action observation), two levels of hemispheric site of TMS (left and right M1), three levels type of conditioning tDCS stimulation factor (cathodal, anodal, sham), and two levels concerning the intervention (pre-tDCS, post-tDCS). Greenhouse-Geisser correction was applied when necessary to compensate for the violation of the assumption of sphericity. In the presence of significant interactions, corrected pairwise comparisons (Bonferroni test) were performed. The level of significance was $p=0.05$.

\section{RESULTS}

The ANOVA on mean MEPs amplitude of the FDI showed an interaction between the four main factors $F_{2,20}=4.700, \mathrm{MSE}=38015.324$, $p=0.021$, while the ANOVA for the ADM muscle did not show any significant effect.

A striking tDCS effect was observed. The same four main factors interaction showed that cathodal tDCS over the right PC produced an inhibitory effect on the corticospinal facilitation induced by the motor imagery task, only for the right M1 TMS, compared to the pre-tDCS condition $(p<0.008)$. Moreover, a significant opposite effect was obtained on the same site (right/ipsilateral M1; $p<0.042$ ), when anodal stimulation was delivered. Sham tDCS did not produce any effect and, as shown in the figure, MEPs' size was almost equal to the pre-tDCS condition (Figure 2A).

In the same line, an effect of cathodal stimulation emerged also for the action observation task. Cathodal tDCS decreased the mean amplitude of MEPs recorded during action observation, selectively when TMS was applied on the right M1, compared to the pretDCS condition ( $p=0.002$; Figure $2 B$ ). No significant effects were observed for anodal and sham stimulation.

An inter-hemispheric effect was observed too: whereas no significant differences emerged between the right and left M1 motorevoked response for sham tDCS, and a slighter but not significant effect was observed for anodal stimulation, cathodal tDCS drastically decreased the corticospinal facilitation of the right/ipsilateral M1 compared to the left M1 $(p<0.001)$, only during the motor imagery task (Figure A1 in Appendix).

Post hoc comparisons highlighted that, regardless of the anodal, cathodal, and sham stimulation, the facilitatory effect of motor imagery on corticospinal output, in terms of increase of MEPs' size, was always higher than that induced by the action observation 


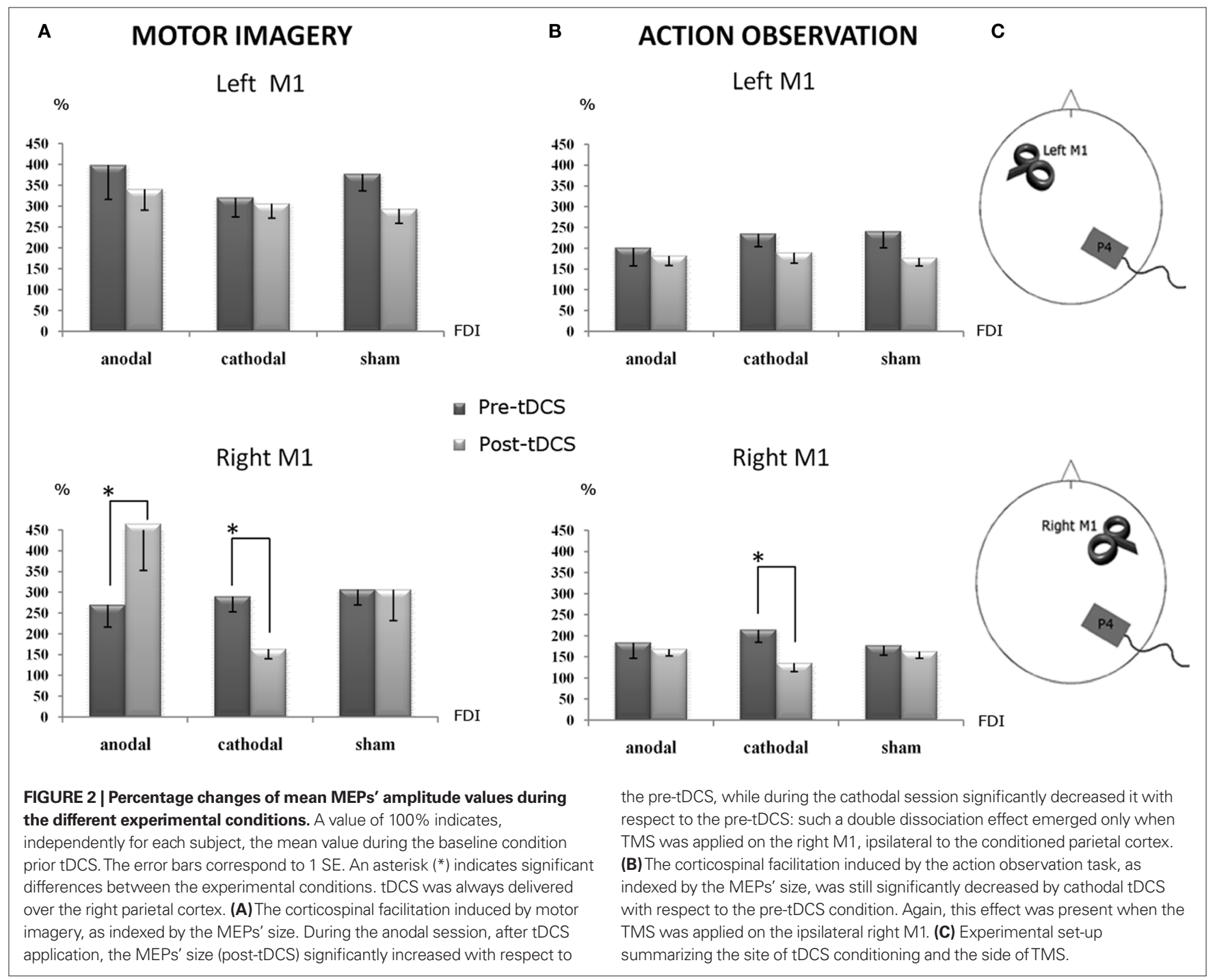

task. This occurred both for the pre-tDCS session and persisted for post-tDCS session during the left M1 TMS stimulation. More specifically, for anodal session, pre-tDCS motor imagery versus pre-tDCS action observation $(p=0.003)$, and tDCS motor imagery versus tDCS action observation $(p=0.011)$. For sham stimulation, pre-tDCS motor imagery versus pre-tDCS action observation $(p=0.011)$ and tDCS motor imagery versus tDCS action observation $(p=0.001)$.

For cathodal stimulation, no significant effects were observed, despite a consistent trend: pre-tDCS motor imagery versus pretDCS action observation $(p=0.112)$ and tDCS motor imagery versus tDCS action observation ( $p=0.059$; Figure 3A).

On the other hand, interesting effects of the tDCS applications, were observed when TMS was delivered on the right M1, thereby ipsilateral to the conditioned parietal site. For anodal session, no effects emerged for pre-tDCS motor imagery versus pre-tDCS action observation, whereas tDCS motor imagery versus tDCS action observation showed a significant effect $(p=0.036)$. For cathodal, only pre-tDCS motor imagery versus pre-tDCS action observation was significant $(p=0.027)$ while tDCS application did not produce any significant effect. For sham, a similar left M1 effects were observed. Interesting interactions emerged both for pre-tDCS motor imagery versus pre-tDCS action observation $(p=0.000)$ and a near significant effect for tDCS motor imagery versus tDCS action observation ( $p=0.063$; Figure 3B). Of note, these data showed that motor imagery has a trend to increase MEPs size with respect to the action observation task, as also indexed by the main effect of "task" factor, $F_{1,10}=21.480 \mathrm{MSE}=1128691.870, p=0.001$.

\section{DISCUSSION}

Human motor control includes a variety of processes such as understanding, planning, imagery, observation, and execution of movements (Mulder, 2007). These are the final responses of a differential brain activity that precedes the experience of an action. PC plays a key role in this sense, since it is implicated as a mediator of all these aspects of motor activities (Singh-Curry and Husain, 2009). On this vein, the present study was aimed to address the role of the right PC in motor imagery and action observation by an accurate 


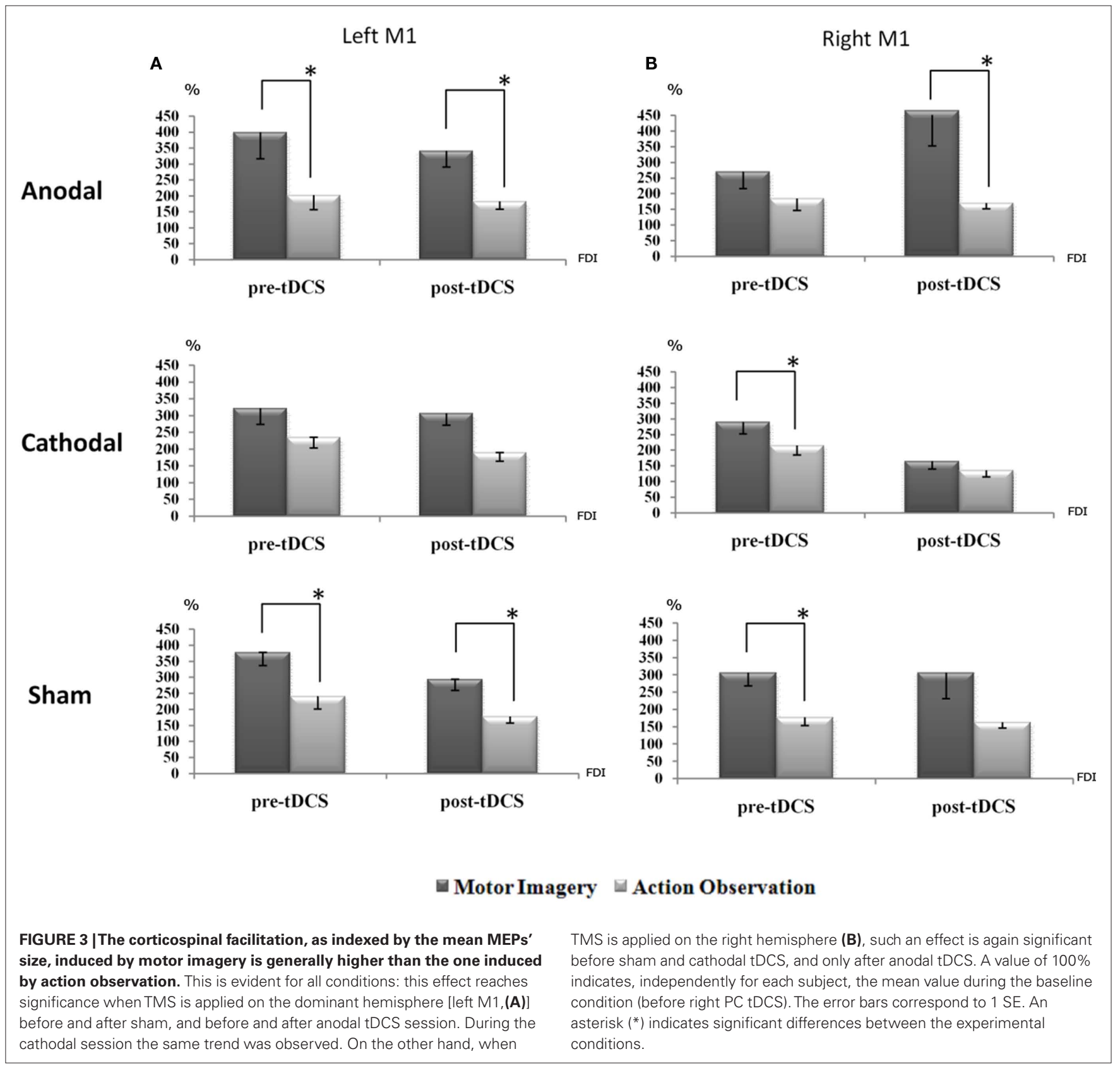

investigation involving, as principle factors of interest, the hemispheric lateralization of the motor response (right and left M1), its off-line conditioning (pre- and post-tDCS), and the effects of such a conditioning on different tasks related to cognitive aspects of motor control, like motor imagery and action observation.

Results clearly show in a causal manner that MEPs' size is consistently higher during motor imagery than during action observation tasks in both hemispheres. This was the case both for pre-tDCS and for anodal, cathodal, and sham tDCS of the right PC, suggesting a more robust corticospinal facilitation induced by motor imagery than by action observation. Such an effect is specific for the FDI muscle, which is the main effector of the imagined/observed actions (i.e., an index/thumb pich grip). Indeed, no significant changes were observed for the ADM muscle, which shares the same cortical representation of the FDI muscle, but is not engaged in pinch grip actions.

Previous studies comparing TMS-induced motor responses during motor imagery and action observation within a unique experimental design, did not find any differences between these two processes in terms of corticospinal output (Patuzzo et al., 2003; Clark et al., 2004; Leonard and Tremblay, 2007). However, it has been recently observed that the corticospinal facilitation induced by motor imagery and action observation is task-dependent, mostly related to the complexity of the imagined, observed or actually executed action (Cattaneo et al., 2009; Roosink and Zijdewind, 2010). Here, we adopted an extremely ecological and simple setting 
requiring a self-triggered imaging and a simple passive action observation task, without goal-directed actions or movements simulation on a computer screen. Therefore, it is plausible that under these circumstances, the adult human motor system may reflect a stronger representation of motor imagery with respect to action observation (Figure 2).

As an extension of this concept, the most striking result of the current study is represented by the efficacy of the right PC tDCS conditioning on the corticospinal excitability of the ipsilateral M1 activity, selectively for the motor imagery task. Anodal stimulation of the right PC definitely increased the right M1 MEPs size, while cathodal stimulation tuned MEPs size down. This confirms the existence of a functional ipsilateral PC-M1 connection subserving not only motor coordination and movement planning (Grefkes and Fink, 2005; Koch et al., 2009), but also motor imagery tasks. This is however, not surprising, since imagery, planning, and execution of actions mostly share the same anatomic-functional substrates (Decety et al., 1994; Parsons et al., 1995; Schnitzler et al., 1997; Hanakawa et al., 2003; Dechent et al., 2004). Moreover, whereas the left M1 excitability was not modulated by right PC tDCS, the right M1 excitability dropped down under cathodal stimulation with respect to the same left M1 (Figure A1 in Appendix), highlighting a significant intra-hemispheric effect mediated by ipsilateral connections between right PC and M1 (Koch et al., 2007, 2008).

On the other hand, the absence of inter-hemispheric effects is apparently in contrast with the observation that a conditioning TMS pulse over the PPC can exert both facilitatory and inhibitory effects on the contralateral M1 excitability (Koch et al., 2009). The most likely explanation should take into account several experimental differences: first, the nature of the required task (Cattaneo et al., 2009; Roosink and Zijdewind, 2010); second, the nature of the conditioning stimulation: tDCS or TMS.

With respect to the nature of the motor task, we have to consider that this "ecological" study did not gaze at tasks involving reaching to an object or observation of goal-directed actions, which are known to engage parieto-motor connections (Koch et al., 2010b). Here, there was no final aim while subjects were imagining or observing an action. This might have reduced the amount of MEPs' facilitation induced by action observation, which commonly involves not only motor and parietal structures, but also the more cognitive ones such as prefrontal cortices (Iacoboni et al., 1999).

As for the nature of conditioning stimulation is concerned, whereas TMS produces a spike-dependent effect that, when applied as paired-pulses as in the Koch's studies (Koch et al., 2007, 2008, 2009) cannot outlast the timing of the first conditioning pulse, tDCS produces bidirectional membrane shift polarization induced by anodal or cathodal tDCS, which is known to outlast the period of stimulation. However, it would be certainly worth to apply the twincoil approach to confirm, in the frame of an "online" approach,

\section{REFERENCES}

Antal, A., Lang, N., Boros, K., Nitsche, M., Siebner, H. R., and Paulus, W. (2008). Homeostatic metaplasticity of the motor cortex is altered during headache-free intervals in migraine with aura. Cereb. Cortex 18, 2701-2705.
Ardolino, G., Bossi, B., Barbieri, S., and Priori, A. (2005). Non-synaptic mechanisms underlie the after-effects of cathodal transcutaneous direct current stimulation of the human brain. J. Physiol. 568, 653-663.

Calvert, G. A. (2001). Crossmodal processing in the human brain: insights

whether motor imagery tasks can engage parieto-motor connections in a similar way to planning, executing and observing actions (Koch et al., 2007, 2008, 2010b). Moreover, tDCS is less focal than TMS application on a target cortical region (Wagner et al., 2007). Thus, we might even consider that the widespread effects of tDCS could have affected the entire parietal-motor-premotor network, although the electrodes montage that we adopted here, should have minimized the spread of stimulation (Cogiamanian et al., 2007; Priori et al., 2008).

It is intriguing that $\mathrm{tDCS}$ of the parietal cortex did not change the excitability of the motor cortex at rest, ipsi- and contralaterally to its application. Such a lack of effects may be due to a state-dependent factor of the parieto-motor network: more specifically, the role of the right $\mathrm{PC}$ in motor control, and its susceptibility to neuromodulatory effects of tDCS, may emerge only for specific processes such as motor imagery and, to a lesser extent, action observation, which definitely engage this area. A similar dissociate reactivity to tDCS conditioning due to state-dependency of the motor system has been already described for the premotor-primary motor cortex network (Quartarone et al., 2004): in this case, the decrease of MEP size at rest following cathodal tDCS of the premotor cortex returned to baseline values after $10 \mathrm{~min}$, while MEPs during motor imagery were suppressed for up to $30 \mathrm{~min}$.

These findings confirmed the existence of parieto-motor pathways, which seems to be crucial not only for planning different grasping actions as recently demonstrated in vivo (Koch et al., 2010a), but also for motor imagery and, to a lesser extent, for action observation.

In conclusion, this study shows that neuromodulation of the right PC with tDCS mainly affects the cortico-cortical connectivity with the ipsilateral M1 in a strictly selective and bidirectional manner when motor imagery and action observation processes are in progress. Current results suggest that conditioning the excitability of the PC by tDCS during cognitive activities linked with motor behavior may represent an alternative approach to modulate the excitability of motor areas. Such a neuromodulatory strategy based on PC-M1 connectivity might be worth to be exploited in rehabilitative settings: for instance, anodal tDCS of the PC might help to down-regulate the abnormal increase in neuronal firing of the motor cortex usually accompanying overuse syndromes and dystonia (Quartarone et al., 2003), while cathodal tDCS of the PC might help to up-regulate the affected motor cortex in stroke patients with pinch-grip motor deficits.

\section{ACKNOWLEDGMENTS}

This work has been partially supported by the European Commission with the Collaborative Project no. 248587, "The Hand Embodied," within the FP7-ICT-2009-4-2-1 program "Cognitive Systems and Robotics."

from functional neuroimaging studies. Cereb. Cortex 11, 1110-1123.

Cattaneo, L., Caruana, F., Jezzini, A., and Rizzolatti, G. (2009). Representation of goal and movements without overt motor behavior in the human motor cortex: a transcranial magnetic stimulation study.J. Neurosci. 29, 11134-11138.
Clark, S., Tremblay, F., and Ste-Marie, D. (2004). Differential modulation of corticospinal excitability during observation, mental imagery and imitation of hand actions. Neuropsychologia 42, 105-112.

Cogiamanian, F., Marceglia, S., Ardolino, G., Barbieri, S., and Priori, A. (2007) 
Improved isometric force endurance after transcranial direct current stimulation over the human motor cortical areas. Eur. J. Neurosci. 26, 242-249.

Cohen, Y. E., and Andersen, R. A. (2002). A common reference frame for movement plans in the posterior parietal cortex. Nat. Rev. Neurosci. 3, 553-562.

Colby, C. L., and Goldberg, M. E. (1999). Space and attention in parietal cortex. Annu. Rev. Neurosci. 22, 319-349.

Decety, J., Grezes, J., Costes, N., Perani, D., Jeannerod, M., Procyk, E. Grassi, F., and Fazio, F. (1997). Brain activity during observation of actions. Influence of action content and subject's strategy. Brain 120(Pt 10), 1763-1777.

Decety, J., Perani, D., Jeannerod, M., Bettinardi, V., Tadary, B., Woods, R., Mazziotta, J. C., and Fazio, F. (1994). Mapping motor representations with positron emission tomography. Nature 371, 600-602.

Dechent, P., Merboldt, K. D., and Frahm, J. (2004). Is the human primary motor cortex involved in motor imagery? Brain Res. Cogn. Brain Res. 19, 138-144.

Fadiga, L., Fogassi, L., Pavesi, G., and Rizzolatti, G. (1995). Motor facilitation during action observation: a magnetic stimulation study. $J$. Neurophysiol. 73, 2608-2611.

Fleming, M. K., Stinear, C. M., and Byblow, W. D. (2010). Bilateral parietal cortex function during motor imagery. Exp. Brain Res. 201, 499-508.

Fogassi, L., and Luppino, G. (2005). Motor functions of the parietal lobe. Curr. Opin. Neurobiol. 15, 626-631.

Giovannelli, F., Silingardi, D., Borgheresi, A., Feurra, M., Amati, G., Pizzorusso, T. Viggiano, M. P., Zaccara, G., Berardi, N., and Cincotta, M. (2010). Involvement of the parietal cortex in perceptual learning (Eureka effect): an interference approach using rTMS. Neuropsychologia 48, 1807-1812.

Grafton, S. T., Arbib, M. A., Fadiga, L., and Rizzolatti, G. (1996). Localization of grasp representations in humans by positron emission tomography 2 . Observation compared with imagination. Exp. Brain Res. 112, 103-111.

Grefkes, C., and Fink, G. R. (2005). The functional organization of the intraparietal sulcus in humans and monkeys. J. Anat. 207, 3-17.

Hanakawa, T., Immisch, I., Toma, K., Dimyan, M. A., van, G. P., and Hallett, M. (2003). Functional properties of brain areas associated with motor execution and imagery. J. Neurophysiol. 89, 989-1002.

Iacoboni, M., Woods, R. P., Brass, M., Bekkering, H., Mazziotta, J. C., and Rizzolatti, G. (1999). Cortical mechanisms of human imitation. Science 286, 2526-2528.
Jeannerod, M. (1995). Mental imagery in the motor context. Neuropsychologia $33,1419-1432$.

Jeannerod, M. (2001). Neural simulation of action: a unifying mechanism for motor cognition. Neuroimage 14, S103-S109.

Koch, G., Cercignani, M., Pecchioli, C., Versace, V., Oliveri, M., Caltagirone, C. Rothwell, J., and Bozzali, M. (2010a). In vivo definition of parieto-motor connections involved in planning of grasping movements. Neuroimage 51, 300-312.

Koch, G., Versace, V., Bonni, S., Lupo, F., Lo, G. E., Oliveri, M., and Caltagirone, C. (2010b). Resonance of cortico-cortical connections of the motor system with the observation of goal directed grasping movements. Neuropsychologia 48 , 3513-3520.

Koch, G., Fernandez del Olmo, M., Cheeran, B., Ruge, D., Schippling, S., Caltagirone, C., and Rothwell, J. C. (2007). Focal stimulation of the posterior parietal cortex increases the excitability of the ipsilateral motor cortex. J. Neurosci. 27, 6815-6822.

Koch, G., Fernandez del Olmo, M., Cheeran, B., Schippling, S., Caltagirone, C., Driver, J., and Rothwell, J. C. (2008). Functional interplay between posterior parietal and ipsilateral motor cortex revealed by twin-coil transcranial magnetic stimulation during reach planning toward contralateral space. J. Neurosci. 28, 5944-5953.

Koch, G., Ruge, D., Cheeran, B., Fernandez del Olmo, M., Pecchioli, C., Marconi, B., Versace, V., Lo Gerfo, E., Torriero, S., Oliveri, M., Caltagirone, C., and Rothwell, J.C. (2009). TMS activation of interhemispheric pathways between the posterior parietal cortex and the contralateral motor cortex. J. Physiol. 587, 4281-4292.

Law, C. T., and Gold, J. I. (2008). Neural correlates of perceptual learning in a sensory-motor, but not a sensory, cortical area. Nat. Neurosci. 11, 505-513.

Leonard, G., and Tremblay, F. (2007). Corticomotor facilitation associated with observation, imagery and imitation of hand actions: a comparative study in young and old adults. Exp. Brain Res. 177, 167-175.

Lotze, M., and Cohen, L. G. (2006). Volition and imagery in neurorehabilitation. Cogn. Behav. Neurol. 19, 135-140.

McCreery, D. B., Agnew, W. F., Yuen, T. G., and Bullara, L. (1990). Charge density and charge per phase as cofactors in neural injury induced by electrical stimulation. IEEE Trans. Biomed. Eng. 37, 996-1001.

Mulder, T. (2007). Motor imagery and action observation: cognitive tools for rehabilitation. J. Neural Transm. 114, 1265-1278.

Nelissen, K., Luppino, G., Vanduffel, W., Rizzolatti, G., and Orban, G.A. (2005). Observing others: multiple action representation in the frontal lobe. Science 310, 332-336.

Nitsche, M. A., Liebetanz, D., Lang, N., Antal, A., Tergau, F., and Paulus, W. (2003a). Safety criteria for transcranial direct current stimulation (tDCS) in humans. Clin. Neurophysiol. 114 2220-2222.

Nitsche, M.A., Nitsche, M. S., Klein, C. C. Tergau, F., Rothwell, J. C., and Paulus, W. (2003b). Level of action of cathoda DC polarisation induced inhibition of the human motor cortex. Clin. Neurophysiol. 114, 600-604.

Nitsche, M. A., Schauenburg, A., Lang, N., Liebetanz, D., Exner, C., Paulus, W., and Tergau, F. (2003c). Facilitation of implicit motor learning by weak transcranial direct current stimulation of the primary motor cortex in the human. J. Cogn. Neurosci. 15, 619-626.

Nitsche, M. A., and Paulus, W. (2000). Excitability changes induced in the human motor cortex by weak transcranial direct current stimulation. $J$. Physiol. 527(Pt 3), 633-639.

Nitsche, M. A., and Paulus, W. (2001) Sustained excitability elevations induced by transcranial DC motor cortex stimulation in humans. Neurology 57, 1899-1901.

Parsons, L. M., Fox, P. T., Downs, J. H., Glass, T., Hirsch, T. B., Martin, C. C., Jerabek, P. A., and Lancaster, J. L. (1995). Use of implicit motor imagery for visual shape discrimination as revealed by PET. Nature 375, 54-58.

Patuzzo, S., Fiaschi, A., and Manganotti, P. (2003). Modulation of motor cortex excitability in the left hemisphere during action observation: a single- and paired-pulse transcranial magnetic stimulation study of self- and non-selfaction observation. Neuropsychologia 41, 1272-1278.

Priori, A., Berardelli, A., Rona, S. Accornero, N., and Manfredi, M. (1998). Polarization of the human motor cortex through the scalp. Neuroreport 9, 2257-2260.

Priori, A., Mameli, F., Cogiamanian, F. Marceglia, S., Tiriticco, M., MrakicSposta, S., Ferrucci, R., Zago, S., Polezzi, D., and Sartori, G. (2008). Lie-specific involvement of dorsolateral prefrontal cortex in deception. Cereb. Cortex 18, 451-455.

Quartarone, A., Bagnato, S., Rizzo, V., Siebner, H. R., Dattola, V., Scalfari, A., Morgante, F., Battaglia, F., Romano, M., and Girlanda, P. (2003). Abnormal associative plasticity of the human motor cortex in writer's cramp. Brain 126, 2586-2596.
Quartarone, A., Morgante, F., Bagnato, S., Rizzo, V., Sant'Angelo, A., Aiello, E., Reggio, E., Battaglia, F., Messina, C., and Girlanda, P. (2004). Long lasting effects of transcranial direct current stimulation on motor imagery. Neuroreport 15, 1287-1291.

Rizzolatti, G., and Craighero, L. (2004). The mirror-neuron system. Annu. Rev. Neurosci. 27, 169-192.

Rizzolatti, G., and Fadiga, L. (1998). Grasping objects and grasping action meanings: the dual role of monkey rostroventral premotor cortex (area F5). Novartis. Found. Symp. 218, 81-95.

Rizzolatti, G., Fogassi, L., and Gallese, V. (2001). Neurophysiological mechanisms underlying the understanding and imitation of action. Nat. Rev. Neurosci. 2, 661-670.

Roosink, M., and Zijdewind, I. (2010). Corticospinal excitability during observation and imagery of simple and complex hand tasks: implications for motor rehabilitation. Behav. Brain Res. 213, 35-41.

Rossi, S., De, C. A., Pasqualetti, P., Ulivelli, M., Fadiga, L., Falzarano, V., Bartalini, S., Passero, S., Nuti, D., and Rossini, P. M. (2008). Distinct olfactory crossmodal effects on the human motor system. PLoS ONE 3, e1702. doi: 10.1371/journal.pone.0001702

Rossi, S., Hallett, M., Rossini, P. M., and Pascual-Leone, A. (2009). Safety, ethical considerations, and application guidelines for the use of transcranial magnetic stimulation in clinical practice and research. Clin. Neurophysiol. 120, 2008-2039.

Rossi, S., Pasqualetti, P., Zito, G., Vecchio, F., Cappa, S. F., Miniussi, C., Babiloni, C., and Rossini, P.M. (2006). Prefrontal and parietal cortex in human episodic memory: an interference study by repetitive transcranial magnetic stimulation. Eur. J. Neurosci. 23, 793-800.

Rossini, P. M., Rossi, S., Pasqualetti, P., and Tecchio, F. (1999). Corticospinal excitability modulation to hand muscles during movement imagery. Cereb. Cortex 9, 161-167.

Ruby, P., and Decety, J. (2003). What you believe versus what you think they believe: a neuroimaging study of conceptual perspective-taking. Eur. J. Neurosci. 17, 2475-2480.

Schnitzler, A., Salenius, S., Salmelin, R., Jousmaki, V., and Hari, R. (1997). Involvement of primary motor cortex in motor imagery: a neuromagnetic study. Neuroimage 6, 201-208.

Singh-Curry, V., and Husain, M. (2009). The functional role of the inferior parietal lobe in the dorsal and ventral stream dichotomy. Neuropsychologia 47, 1434-1448.

Starr, A., Caramia, M., Zarola, F., and Rossini, P.M.(1988). Enhancement of 
motor cortical excitability in humans by non-invasive electrical stimulation appears prior to voluntary movement. Electroencephalogr. Clin. Neurophysiol. 70, 26-32.

Vandermeeren, Y., Jamart, J., and Ossemann, M. (2010). Effect of tDCS with an extracephalic reference electrode on cardio-respiratory and autonomic functions. BMC Neurosci. 11, 38. doi: 10.1186/1471-2202-11-38

Wagner, T., Valero-Cabre, A., and PascualLeone, A. (2007). Noninvasive human brain stimulation. Annu. Rev. Biomed. Eng. 9, 527-565.

Wassermann, E. M., and Grafman, J. (2005). Recharging cognition with DC brain polarization. Trends Cogn. Sci. 9, 503-505.

Zimmer, H. D. (2008). Visual and spatial working memory: from boxes to networks. Neurosci. Biobehav. Rev.32, 1373-1395.

Conflict of Interest Statement: The authors declare that the research was conducted in the absence of any commercial or financial relationships that could be construed as a potential conflict of interest.

Received: 16 June 2011; paper pending published: 12 July 2011; accepted: 07 August 2011; published online: 30 August 2011.

Citation: Feurra M, Bianco G, Polizzotto NR, Innocenti I, Rossi A and Rossi S (2011) Cortico-cortical connectivity between right parietal and bilateral primary motor cortices during imagined and observed actions: a combined TMS/tDCS study. Front. Neural Circuits 5:10. doi: 10.3389/ fncir.2011.00010

Copyright (c) 2011 Feurra, Bianco, Polizzotto, Innocenti, Rossi and Rossi. This is an open-access article subject to a nonexclusive license between the authors and Frontiers Media SA, which permits use, distribution and reproduction in other forums, provided the original authors and source are credited and other Frontiers conditions are complied with. 


\section{APPENDIX}

The possible differences in cortical excitability previous and after tDCS, was addressed by six separate repeated measures one-way ANOVAs (left and right M1 TMS for each tDCS session), each one with a two-levels factor intervention (pre- and post-tDCS).

ANOVA $\mathrm{n} 1$ for anodal session and right M1 TMS; $\mathrm{n} 2$ for anodal session and left M1 TMS; $\mathrm{n} 3$ for cathodal session and right M1 TMS; $\mathrm{n} 4$ for cathodal session and left M1 TMS; 5 for sham session and right M1 TMS; $\mathrm{n} 6$ for sham session and left M1 TMS). Greenhouse-Geisser correction was applied when necessary to compensate for the violation of the assumption of sphericity. In the presence of significant interactions, corrected pairwise comparisons (Bonferroni test) were performed. The level of significance was $p=0.05$.

The six preliminary ANOVAs did not show any significant effect of the intervention factor $\left[\mathrm{n} 1,\left(F_{1,10}=0.045, \mathrm{MSE}=3387.682\right.\right.$ $p=0.836) ; \mathrm{n} 2,\left(F_{1,10}=1.053, \operatorname{MSE}=154393.136, p=0.329\right) ; \mathrm{n} 3$ $\left(F_{1,10}=2.653, \mathrm{MSE}=269288.909 p=0.134\right) ; \mathrm{n} 4\left(F_{1,10}=0.135\right.$, $\mathrm{MSE}=24622.545, p=0.721) ; \mathrm{n} 5\left(F_{1,10}=0.151, \mathrm{MSE}=18386.182\right.$ $\left.p=0.706) ; \mathrm{n} 6\left(F_{1,10}=1.037, \mathrm{MSE}=55300.409, p=0.333\right)\right]$.

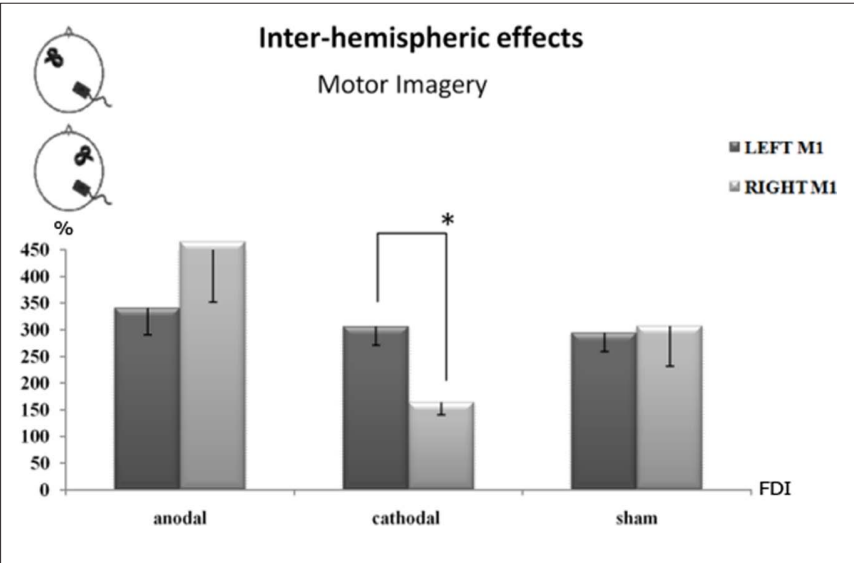

FIGURE A1 | Percentage changes of mean MEPs' amplitude values during the different experimental conditions. A value of $100 \%$ indicates, independently for each subject, the mean value during the baseline condition (before tDCS). The error bars correspond to $1 \mathrm{SE}$. An asterisk (*) indicates significant differences between the experimental conditions. tDCS was always delivered over the right parietal cortex. Cathodal tDCS significantly decreased the corticospinal facilitation induced by motor imagery, as indexed by the mean MEPs' size, when TMS was applied on the right primary motor cortex with respect to the left one. 